\title{
Screening of FDA-approved compound library identifies potential small-molecule inhibitors of SARS-CoV-2 non-structural proteins NSP1, NSP4, NSP6 and NSP13: molecular modeling and molecular dynamics studies
}

\author{
Shobana Sundar ${ }^{1}$. Lokesh Thangamani ${ }^{1}$. Shanmughavel Piramanayagam ${ }^{1}$. Chandrasekar Narayanan Rahul ${ }^{2}$. \\ Natarajan Aiswarya ${ }^{2} \cdot$ Kanagaraj Sekar ${ }^{2} \cdot$ Jeyakumar Natarajan ${ }^{3}$
}

Received: 10 March 2021 / Revised: 8 May 2021 / Accepted: 31 May 2021 / Published online: 9 June 2021

(c) The Author(s), under exclusive licence to Springer Nature Singapore Pte Ltd. 2021

\begin{abstract}
COVID-19, the current global pandemic has caused immense damage to human lives and the global economy. It is instigated by the SARS-CoV-2 virus and there is an immediate need for the identification of effective drugs against this deadly virus. SARS-CoV-2 genome codes for four structural proteins, sixteen non-structural proteins (NSPs) and several accessory proteins for its survival inside the host cells. In the present study, through in silico approaches, we aim to identify compounds that are effective against the four NSPs namely, NSP1, NSP4, NSP6 and NSP13 of SARS-CoV-2. The selection criteria of these four NSP proteins are they are least explored and potential targets. First, we have modeled the 3D structures of these proteins using homology modeling methods. Further, through molecular docking studies, we have screened the FDA-approved compounds against these modeled proteins and reported their docking scores. To gain dynamic insights, molecular dynamics studies have also been carried out for the best scored ligand against the NSPs. This study can further pave way for exposing more number of compounds against these proteins and enhance COVID-19 treatment.
\end{abstract}

Keywords COVID-19 $\cdot$ NSPs $\cdot$ FDA-approved compounds $\cdot$ Molecular docking $\cdot$ Molecular dynamics

\section{Introduction}

Coronavirus disease in 2019 (COVID-19) is the current pandemic threatening the whole world. It is caused by severe acute respiratory syndrome coronavirus 2 (SARS-CoV-2). This highly pathogenic human coronavirus (CoV) was first reported in Wuhan, China, in December 2019. As of April 2021, nearly 141 million people have been affected worldwide and almost 3 million people have died after contracting this deadly virus (WHO 2021). This novel CoV belongs

Jeyakumar Natarajan

n.jeyakumar@yahoo.co.in

1 Computational Biology Lab, Department of Bioinformatics, Bharathiar University, Coimbatore, India

2 Department of Computational and Data Sciences, Indian Institute of Science, Bangalore, Karnataka, India

3 Data Mining and Text Mining Laboratory, Department of Bioinformatics, Bharathiar University, Coimbatore, Tamil Nadu, India to the genus Betacoronavirus in the family Coronaviridae and has one of the largest known RNA genomes ( $29.7 \mathrm{~kb})$ among RNA viruses, along with SARS-CoV and the Middle East respiratory syndrome coronavirus (MERS-CoV). The genome of SARS-CoV-2 has $82 \%$ sequence identity to SARS-CoV and has a lesser sequence identity to MERS-CoV.

These highly pathogenic $\mathrm{CoVs}$ are enveloped and contain single-stranded RNA betacoronaviruses. The $\sim 30 \mathrm{~kb}$ SARSCoV-2 genomes possess at least six open reading frames (ORFs). The first ORF (ORF1a/b) possesses two-thirds of the whole genome length and encodes the 16 non-structural proteins (NSP1-16). ORFs near the 3'-end of the genome encode four main structural proteins including spike (S), membrane (M), envelope (E), and nucleocapsid (N) proteins. The sixteen non-structural proteins are thought to play a vital role in SARS-CoV-2 replication (Chen et al. 2020). However, the structural proteins are important for virion assembly as well as for causing infection. Additionally, specific structural and accessory proteins are also 
encoded by the SARS-CoV-2 genome. The SARS-CoV-2 infection process is initiated via the viral entry mediated by the interaction of the Spike (S) glycoprotein within the host angiotensin-converting enzyme 2 (ACE2) receptor and cleavage of the $\mathrm{S}$ protein by the host transmembrane serine protease 2 (TMPRSS2) prior to the fusion to the host cell membrane (Gil et al. 2020). Once the virus enters the host cell, it gets disassembled to release the nucleocapsid and the viral genome. The structural proteins and all the sixteen non-structural proteins have an important role in the viral pathogenesis and thus serve as important drug targets against SARS-CoV-2 (Fig. 1).

\section{Current drugs used against NSPs}

All the sixteen NSPs are the druggable target; however, the functions of certain NSPs remain elusive. Certain NSPs like NSP5 (3CLpro) which is the main protease of SARS-CoV-2 are exclusively studied and various studies have proven that many inhibitors like lopinavir and ritonavir are effective and are currently being used to treat COVID-19 patients (Cao et al. 2020). Similarly, RdRp (NSP12) is a RNA-dependent RNA polymerase and is elaborately studied. Drugs like Remdesivir and Favipiravir are widely used against RdRp and are also proved to be effective for the treatment of COVID-19 patients (Wang et al. 2020). Therefore, throughout the globe, the other NSPs are also being tested as drug targets and many studies are going on to find some potential inhibitory compounds against these NSPs.

Computational screening of Food and Drug Administration (FDA)-approved drugs against SARS-CoV-2 is a promising method for the identification of suitable drug molecules that can be used for treatment of COVID-19 patients. Recently, many computational studies investigating potential small molecules against SARS-CoV-2 spike protein, Mpro (NSP5), PLpro (NSP3), or RdRp (NSP12) proteins have been published (Hosseini et al. 2020).

In this study, through in silico approaches, we focus on four NSPs (NSP1, NSP4, NSP6 \& NSP13) of SARS$\mathrm{CoV}-2$, which are least explored and its 3D structures remain unresolved to date. Through this study, we aim to find FDA-approved compounds which acts as a potential inhibitors against these four selected NSPs.

\section{Methods}

\section{Homology modeling}

Protein sequences of the selected NSPs (NSP1, NSP4, NSP6 and NSP13) were retrieved from UniprotKB (ID: P0DTD1) and subjected to homology modeling using SWISS-MODEL server (Schwede et al. 2003). Sequence of NSP6 bearing low similarity to the template structure was modeled using $\mathrm{Ab}$ initio method in ROBETTA server (Rohl et al. 2004). All the homology models were validated using Ramachandran plot in PROCHECK server (Laskowski et al. 2006).

\section{Molecular docking}

\section{Protein preparation and receptor grid generation}

Prior to molecular docking, all the validated homology models were prepared using the Protein Preparation wizard of the Schrodinger suite. OPLS_2005 force field was used to energy minimize the proteins. The grid box was generated for the entire protein residues and blind docking was performed.

\section{Ligand retrieval and preparation}

ZINC database (Irwin et al. 2005) is a public repository containing millions of chemical compounds under diverse subsets such as natural products, chemical inhibitors, FDAapproved compounds etc., which can be downloaded in different molecular formats. The 1615 FDA-approved compounds available in the FDA subset of ZINC database were retrieved and used in this study. All the compounds were downloaded in SMILES format and prepared using LigPrep module of Schrodinger and OPLS_2005 force field was used for energy minimization of the compounds.
Fig. 1 The genome of SARS$\mathrm{CoV}-2$ which encodes the structural (spike (S), membrane $(\mathrm{M})$, envelope (E), and nucleocapsid $(\mathrm{N}))$ proteins and the non-structural proteins (NSPs) are depicted

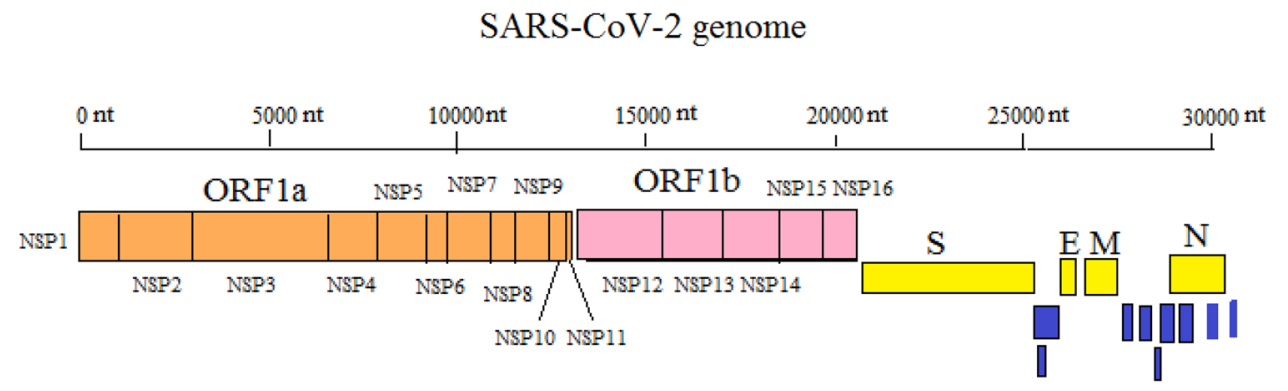

Acessory Proteins 


\section{Molecular docking studies}

The protein-ligand molecular docking studies were performed using the GLIDE module of Schrodinger. Extra Precision (XP) method was employed for docking. The protein-ligand interactions were visualized using the XP visualizer.

\section{Molecular dynamics studies}

Molecular dynamics simulation of the NSP proteins-ligand docked complexes was performed using GROMACS version 2020 (Abraham et al. 2015). GROMOS 43a1 force field was employed for simulations. Single point charge (SPC) water model was used to solvate the protein laid in a cubic box with a distance of $2 \mathrm{~nm}$ from the box to the surface of the protein. The systems were neutralized by adding the corresponding ions. Energy minimization of the docked complexes was carried out using the steepest descent algorithm. Equilibration of the systems was carried out using NVT and NPT ensembles for $100 \mathrm{ps}$ each. The V-rescale thermostat was used for equilibration with a reference temperature of $300 \mathrm{~K}$. Finally, the production MD run was carried out for $25 \mathrm{~ns}$ with a time-step of $2 \mathrm{fs}$. Coordinates of the docked complexes were saved every $10 \mathrm{ps}$ and used for further analysis. Xmgrace was used for plotting graphs.

\section{Results and discussion}

\section{In silico studies on non-structural proteins NSP1, NSP4, NSP6 and NSP13}

Genome of SARS-CoV-2 has about six major ORFs and several accessory proteins. ORF1a and ORF1ab are the two major ORFs which encode the NSPs required for virion replication process. Subsequently, the other ORFs encode for the structural proteins of SARS-CoV-2, namely spike surface glycoprotein $(\mathrm{S})$, envelope protein $(\mathrm{E})$, matrix protein (M), and nucleocapsid protein (N). NSPs are crucial components for the viral replication machinery as they are involved in viral RNA transcription and replication, as well as in impeding the host innate immunity. Studies on various NSP proteins reveal their potential role in viral infection and also help in designing of novel drugs against viral replication and immune evasion machinery. This study examines four less explored NSPs namely, NSP1, NSP4, NSP6 and NSP13 as the potential targets. The detailed molecular modeling, docking and simulation of results of all for selected NSPs are outlined below.

\section{Non-structural protein 1 (NSP1) of SARS-CoV-2}

NSP1 also called as leader protein as it is the first N-terminal cleavage product of the replicase polyprotein (ORF1ab) and is produced by the action of the protease PLpro. It is present in the cytoplasm of the infected cells and it has certain important functions such as host mRNA degradation, suppression of interferon (IFN) expression and host antiviral signaling pathways (Narayanan et al. 2008). NSP1 has important role in viral replication and in regulation of translation process. It is not highly conserved among the other $\beta$-coronaviruses, such as SARS and MERS. NSP1 directly interact with the host ribosome (40S subunit) and ends the host translation by blocking the 40S subunit of the ribosome or it performs an endonucleolytic cleavage near the $5^{\prime}$ UTR of the host mRNA, thus making them ineffectual for translation (Huang et al. 2011). Studies also unravel that NSP1 inhibits the production of host-defense components such as type-I interferons, interleukins, etc., Consequently, NSP1 is considered as an important drug target for COVID-19 treatment. Recent studies illustrated some potential inhibitors for the inhibition of NSP1-viral RNA binding (Sharma et al. 2020; de Lima Menezes et al. 2020).

\section{Homology modeling and validation of NSP1 of SARS-CoV-2}

The protein FASTA sequence of NSP1 of SARS-CoV-2 was retrieved from UniprotKB (ID: P0DTD1, residues 13-127) and was modeled using SWISS-MODEL server. The 3D structure of SARS-CoV NSP1 (PDB ID: 2HSX) which bears $86.09 \%$ sequence identity to the NSP1 of SARS-CoV-2 was used as the template for homology modeling. The homology model of NSP1 of SARS-CoV-2 obtained is depicted in Figure S1a. The NSP1 modeled structure comprises of two helices and five beta strands connected with loops. The Ramachandran plot which was used for the validation of the homology model shows that the homology model of NSP1 of SARS-CoV-2 shows that nearly $83.19 \%$ of the residues are in the favored region (Fig. S1b). ERRAT score for the NSP1 homolog model was 89.7959 which indicates the model is good for further studies. While preparing this manuscript, Clark et al. (2021) have published the 3D protein structure of NSP1. The RMSD was computed between the homology model and the 3D structure of NSP1, and it was around $0.8 \AA$. RMSD values less than $3 \AA$ can be considered for closely homologous proteins (Reva et al. 1998).

\section{Molecular docking studies of NSP1 against the FDA-approved compounds}

In this study, retrieved 1615 FDA-approved compounds from ZINC database were made to dock with the NSP1 of SARS-CoV-2 using the GLIDE module of Schrodinger. 
Ceftolozane (ZINC000009164421), a semi-synthetic, broad-spectrum, fifth-generation cephalosporin antibiotic (Duin and Bonomo 2016), was found to have high affinity against NSP1 and it had a docking score of $-8.135 \mathrm{kcal} /$ mol. Ceftolozane interactions with NSP1 are depicted in Fig. 2a, b, respectively. As NSP1 is said to interact with the host mRNA and degrade it, its site of interaction with the mRNA is the region which can be targeted by the inhibitors. Previously, the analysis of the NSP1 structure of SARS-CoV reveals that the positively charged residues like K48, R125 and K126 are probably interacting with mRNA (Sakai et al. 2017). Similarly, from our docking studies, Ceftolozane was found in proximity with the positively charged residues like R43, R124 and K125 of the NSP1 of the SARS-CoV-2 and may inhibit the viral RNA binding.

\section{Molecular dynamics simulation analysis of NSP1- ceftolozane docked complex}

MD analysis reveals that NSP1 was stable from $3 \mathrm{~ns}$ till the end of simulation (Fig. 3). Radius of gyration (Rg) indicates the compactness of a protein structure throughout the dynamics. Rg graph of NSP1 also indicates that the complex is very stable throughout the dynamics (Fig. 4). Regions having fluctuations with RMSF $>0.2 \mathrm{~nm}$ are considered to be mobile and have a significant role in protein function. RMSF graph of NSP1 obtained during dynamics is depicted in Fig. 5a. Residues 75-81 are a part of $\beta$-hairpin connecting two anti-parallel $\beta$-strands $\beta 3$ and $\beta 4$ moves away from the ligand-binding site (Fig. 5b). The hydrogen bonds obtained during dynamics between NSP1 and Ceftolozane are depicted in Fig. 6.

\section{Molecular docking studies of NSP1 with PKP2}

Gordon et al. 2020 have found certain protein-protein interactions of NSP1 and we performed protein-protein docking studies of NSP1 with Plakophilin-2 (PKP2) using Cluspro server. We have found interactions between NSP1 and PKP2 and are depicted in Fig. 7. PKP2 interactions with NSP1 were found to be in proximal with the Ceftolozane-binding site (Fig. 7).
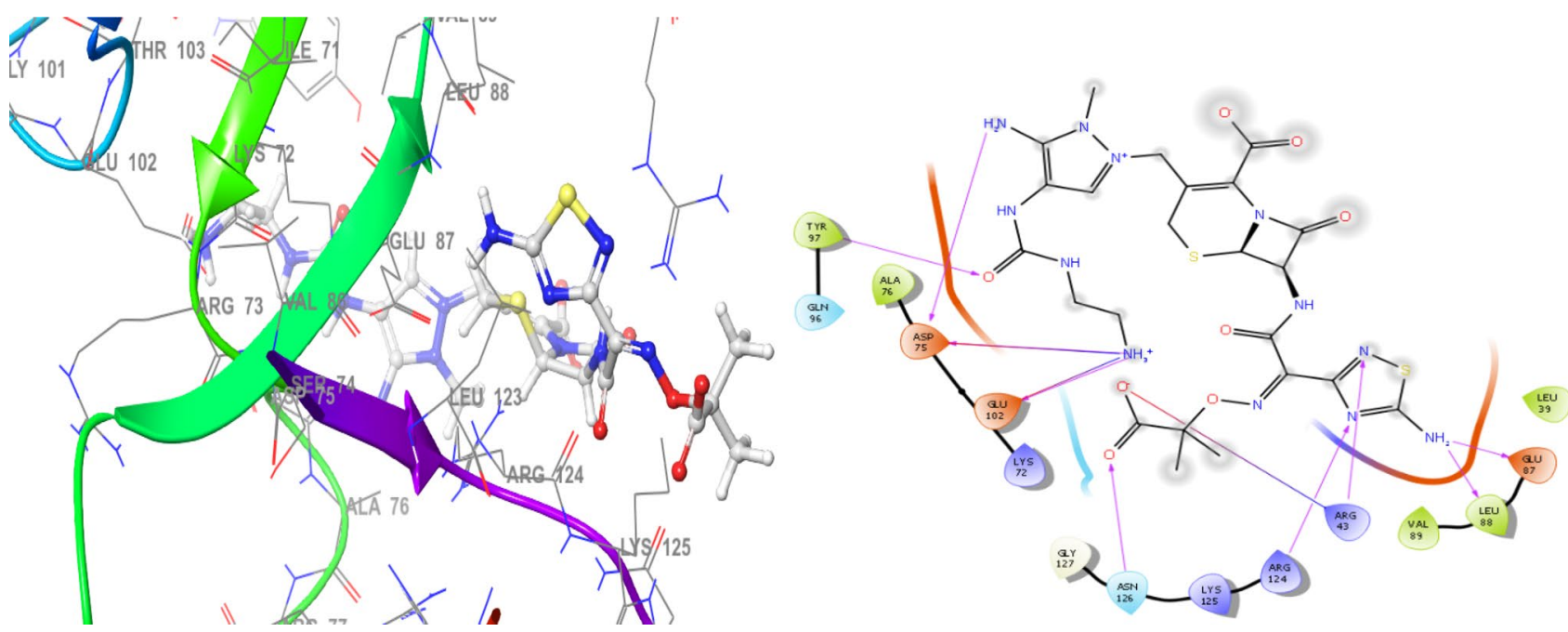

Fig. 2 Ceftolozane interactions with modeled NSP1. Hydrogen bonds between NSP1 and ligand are depicted in the figures

Fig. 3 RMSD graphs of NSP1, NSP4, NSP6 and NSP13

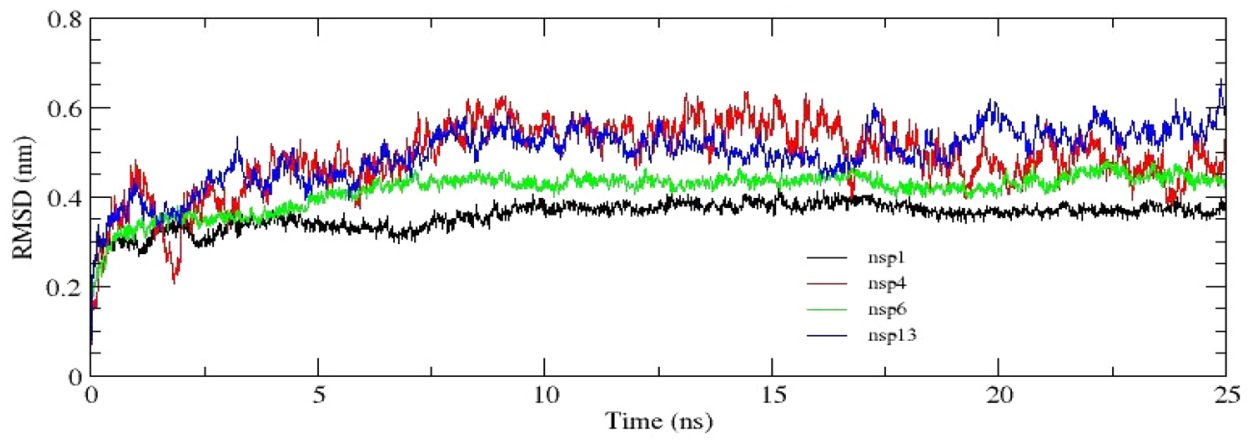


Fig. 4 Rg graphs of NSP1, NSP4, NSP6 and NSP13
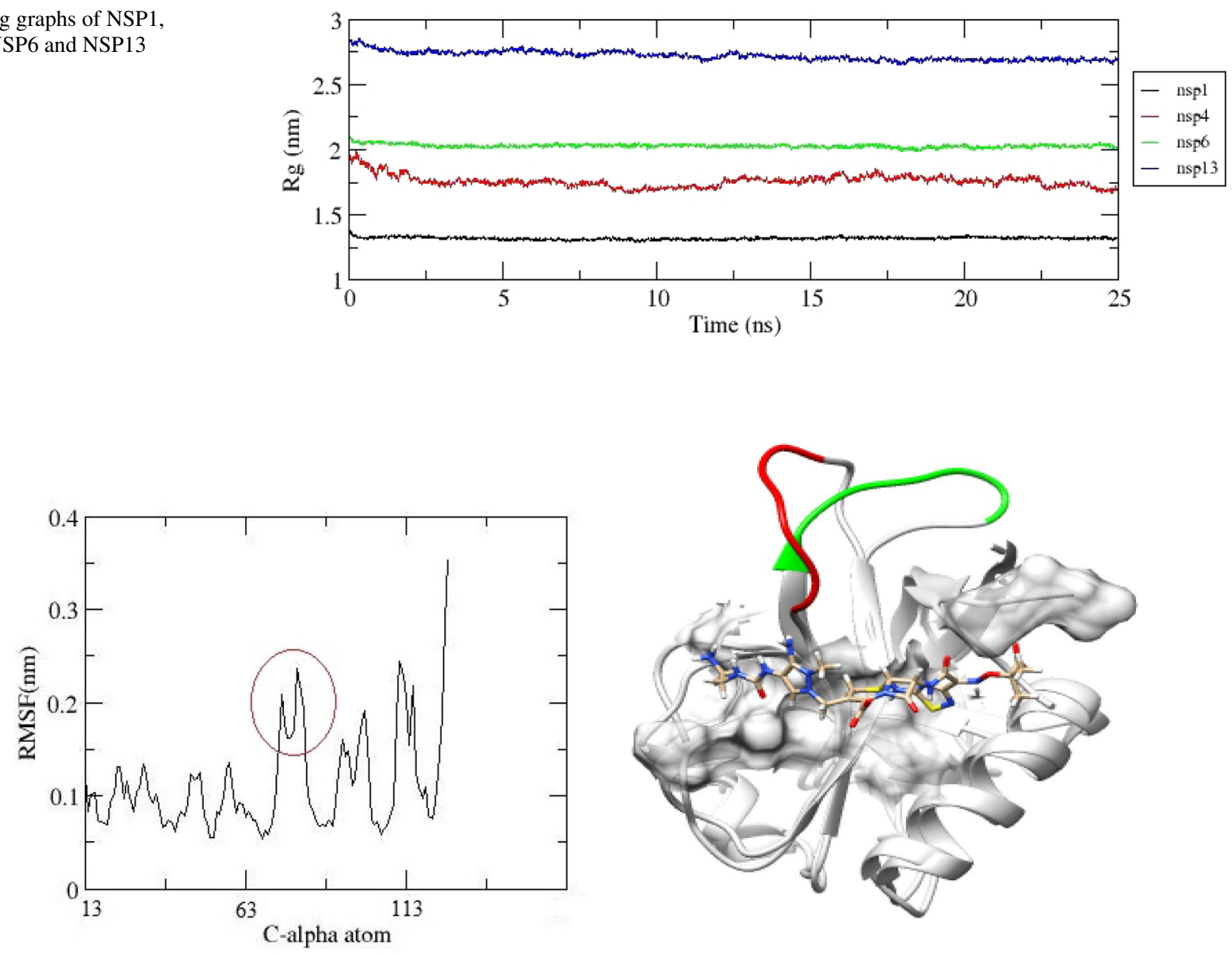

Fig. 5 Fluctuations of NSP1 residues during dynamics. a Red circle in indicates the residues $75-81$ b Superimposed figure of NSP1 at 0 ns and at $25 \mathrm{~ns}$, dynamics of the residues $75-81$ ( $0 \mathrm{~ns}$ in red; $25 \mathrm{~ns}$ in green) is shown, Ceftolozane is represented as atoms

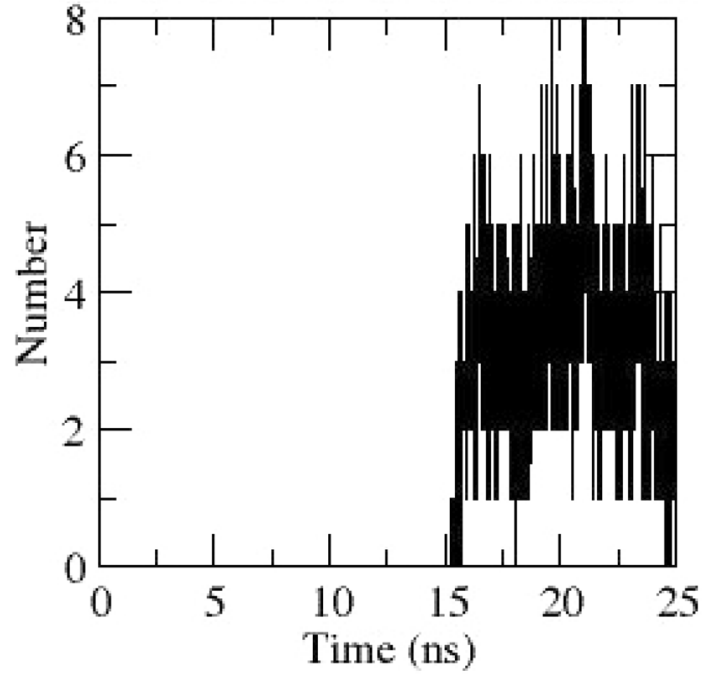

Fig. 6 Hydrogen bonds obtained during dynamics between NSP1 and Ceftolozane

\section{Non-structural protein 4 (NSP4) of SARS-CoV-2}

Generally, it is believed that the SARS-CoV-2 replication takes places on the cytoplasmic double membrane vesicles (DMVs) originated from the endoplasmic reticulum of the infected cells. Many studies on different RNA viruses have shown that the replication complexes are linked with cellular membrane by the hydrophobic domains from the non-structural proteins. Of the 16 SARS-CoV-2 NSPs, only NSP3, NSP4 and NSP6 are known to possess transmembrane domains and are proposed to be involved in the assembly of virally-induced cytoplasmic DMVs which is necessary for viral replication. Sakai et al. in 2017 has demonstrated that the membrane rearrangement induced by the NSP3-NSP4 interaction is crucial for the optimal replication of SARS-CoV. Membrane topology studies of SARS NSP4 have revealed that both the amino and the carboxy termini are exposed to the cytoplasm and further 
Fig. 7 Protein-protein

molecular docking studies of NSP1 with PKP2. Interactions between NSP1 and PKP2 are also depicted
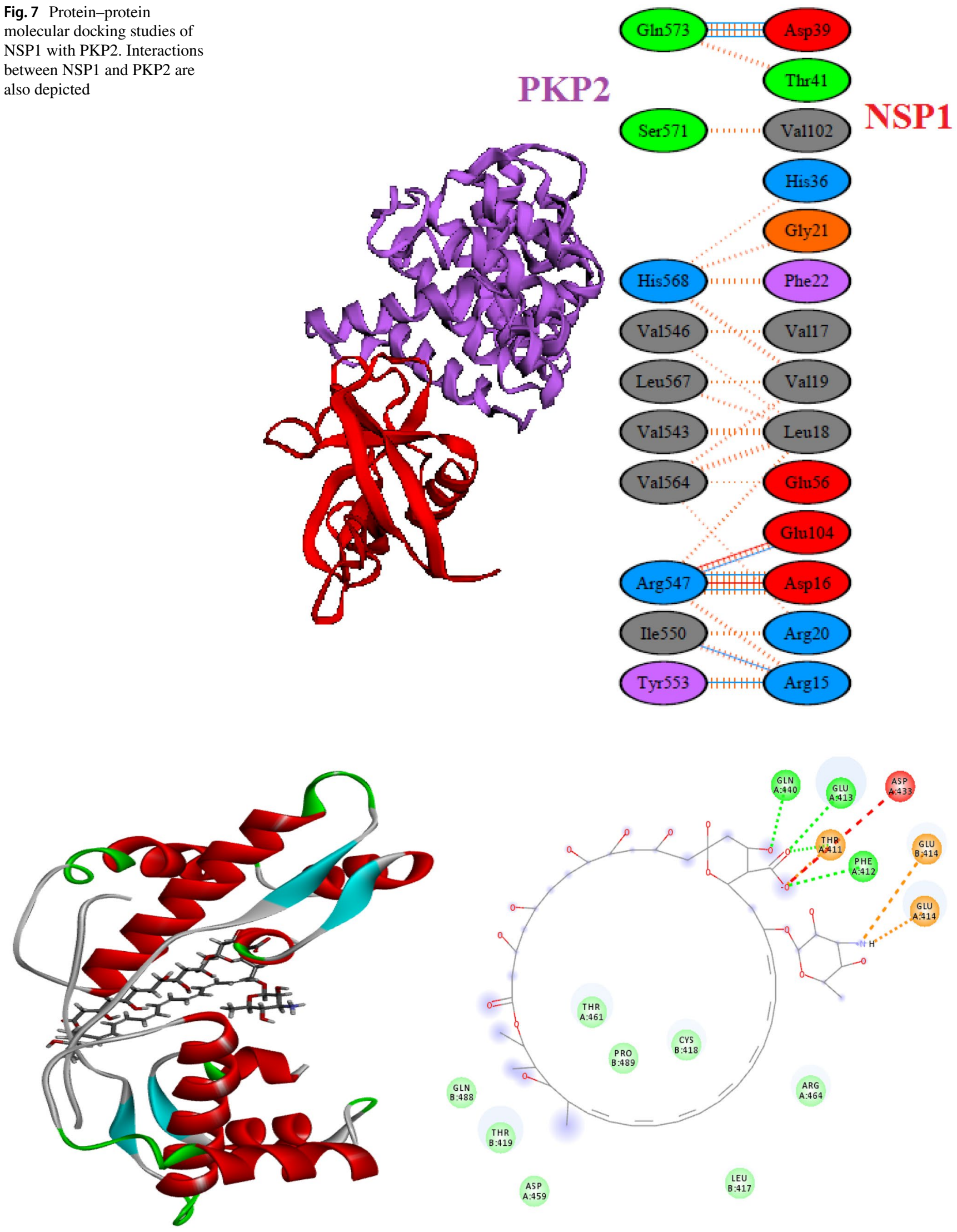

Fig. 8 Amphotericin B interactions with modeled NSP4. Hydrogen bonds between NSP4 and Amphotericin B are depicted in the figures 
they show that all the four hydrophobic domains of NSP4 are membrane spanning. Moreover, a strong immunolabeling of the NSP4 C-terminus residues was observed on the DMVs from the SARS-CoV-infected cells which suggest that it may play an important role in the coronavirus replication complex assembly (Xu et al. 2009).

\section{Homology modeling of the C-terminus region of NSP4 of SARS-CoV-2}

The C-terminal protein FASTA sequence of NSP4 of SARS-CoV-2 was retrieved from UniprotKB (ID: P0DTD1, residues 3175-3263 (411-499)) and was modeled using SWISS-MODEL server. The 3D structure of the C425S mutant C-terminal region of NSP4 (PDB ID: $3 \mathrm{VCB}$ ) from mouse hepatitis virus A59, which bears $61.36 \%$ sequence identity to the $\mathrm{C}$-terminus region of NSP4 of SARS-CoV-2 was used as the template for homology modeling. The homology model of the C-terminus NSP4 of SARS-CoV-2 obtained is a homodimer and is depicted in Figure S2a. The Ramachandran plot which was used for the validation of the homology model shows that the homology model of the C-terminus NSP4 of SARS$\mathrm{CoV}-2$ shows that nearly $95.4 \%$ of the residues are in the favored region (Fig. S2b). Moreover, ERRAT score for the NSP4 homolog model was 92.9577 which indicates the model is good for further studies.

\section{Molecular docking studies of NSP4 against the FDA-approved compounds}

FDA-approved compounds were made to dock with the C-terminal region of NSP4 homology model of SARSCoV-2 using the GLIDE module of Schrodinger. Amphotericin B (ZINC000253387843) was found to have high affinity against the C-terminal region of NSP4 and it had a docking score of $-9.268 \mathrm{kcal} / \mathrm{mol}$. Generally, Amphotericin $B$ shows a high order in vitro activity against many species of fungi (Lemke et al. 2005). Amphotericin B interactions with modeled C-terminal region of NSP4 are depicted in Fig. 8.

\section{Molecular dynamics simulation analysis of NSP4- Amphotericin B docked complex}

MD analysis reveals that NSP4 was stable around $15 \mathrm{~ns}$ till the end of simulation (Fig. 3). Rg graph of NSP4 drops down from $1.9 \mathrm{~nm}$ approx to $1.7 \mathrm{~nm}$, indicating that NSP4 would have attained a more compact structure during dynamics (Fig. 4). RMSF graph of NSP4 obtained during dynamics is depicted in Fig. 9. Residues 420-440 comprise a short $\alpha$-helix and loops; residues $480-486$ consist of a loop between an helix and a strand having fluctuations above $0.2 \mathrm{~nm}$ (Fig. 9). Both of these regions tend to move towards the ligand-binding site and trying to occlude the cavity, resulting in a closed conformation during the dynamics.
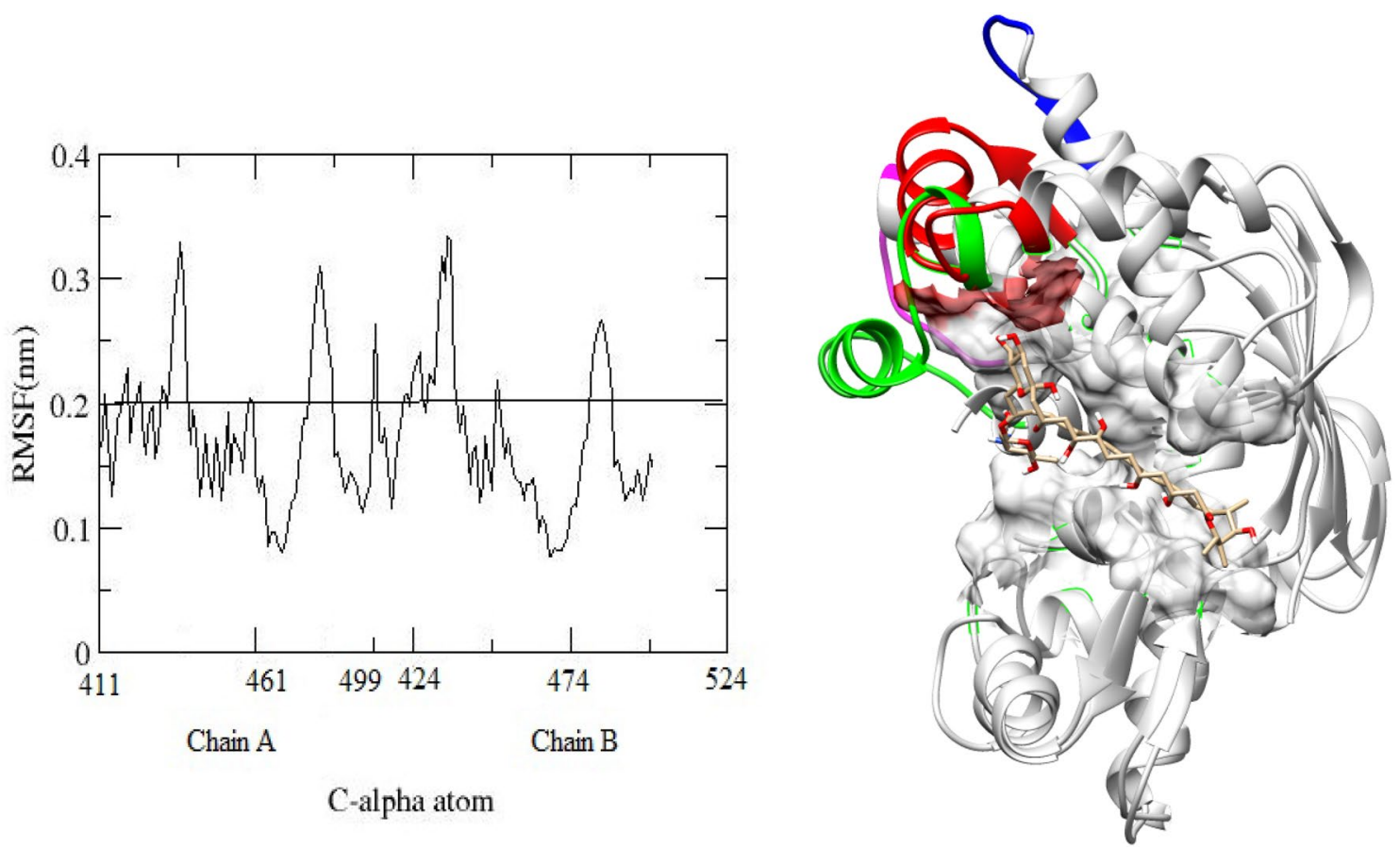

Fig. 9 Fluctuations of NSP4 residues during dynamics. Superimposed figure of NSP1 at 0 ns and at 25 ns, dynamics of the residues $420-440$ (0th ns in red; 25th ns in green) is shown, residues 480-486 (0th ns in blue; 25th ns in pink). Amphotericin B is represented as atoms 


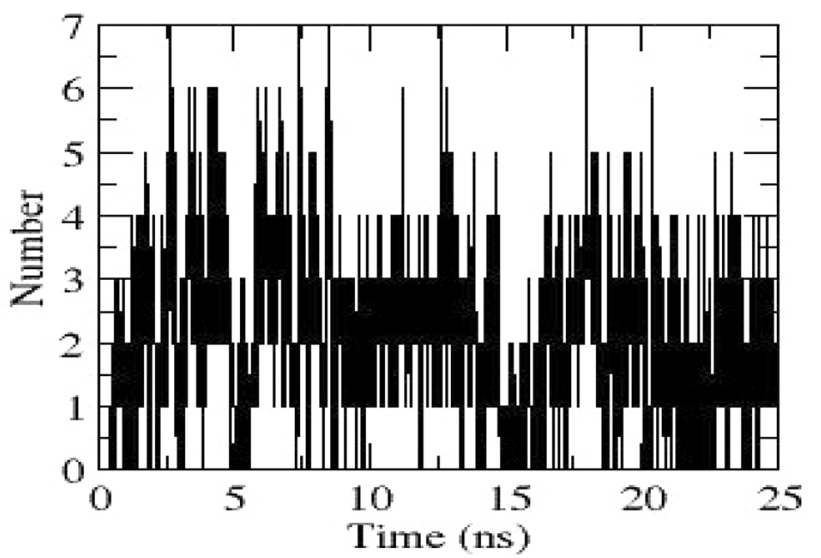

Fig. 10 Hydrogen bonds obtained between NSP4 and Amphotericin B through dynamics
The hydrogen bonds between NSP4 and Amphotericin B obtained through dynamics are depicted in Fig. 10.

\section{Protein-protein molecular docking studies of NSP4 with IDE}

Protein-protein docking studies of NSP4 with Insulindegrading enzyme (IDE) were carried out using Cluspro server. Interactions of NSP4 with IDE are depicted in Fig. 11. From the interaction figures of IDE and Amphotericin, we found that both of them bound to the same binding site of NSP4.

\section{Non-structural protein 6 (NSP6) of SARS-CoV-2}

NSP6 is located in the endoplasmic reticulum (ER) and produces autophagosomes. Positive strand RNA viruses like SARS, MERS, SARS-CoV-2 generate autophagosomes for facilitation of assembly of replicase proteins. NSP6 along

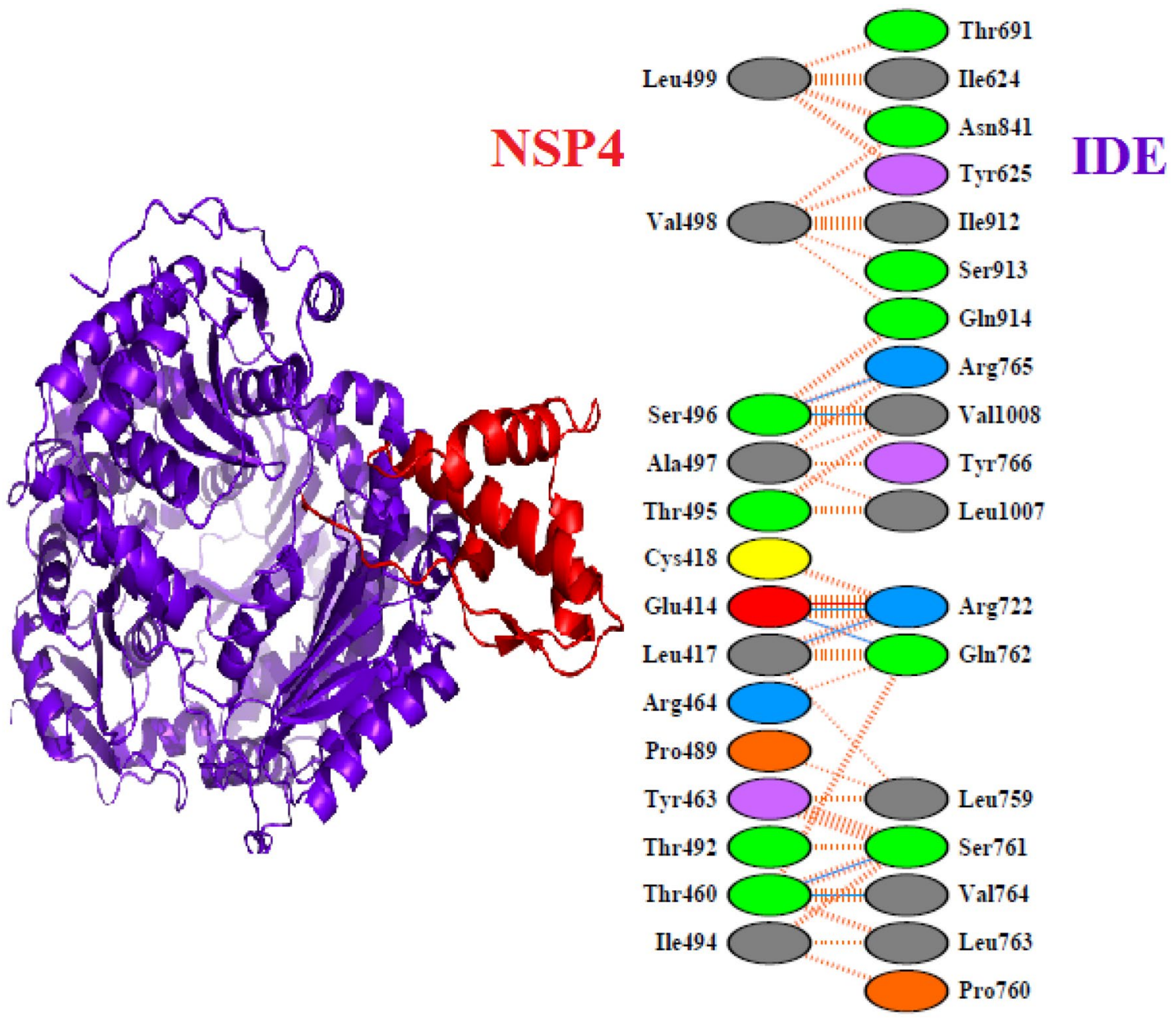

Fig. 11 Protein-protein molecular docking studies of NSP4 with IDE. Interactions between NSP4 and IDE are also depicted 
with NSP3 and NSP4 are involved in the formation of replication complexes which play an important role in viral infection. It has also been revealed that NSP6 may favor coronavirus infection by limiting the autophagosomes to deliver viral components to lysosomes for degradation (Benvenuto et al. 2020).

\section{Homology modeling of NSP6 of SARS-CoV-2}

FASTA sequence of NSP6 of SARS-CoV-2 was retrieved from UniprotKB (ID: P0DTD1, residues 3570-3859 (1-290)) and was modeled using ROSETTA server. Due to unavailability of the homolog structure for NSP6 in the PDB database, ab initio rosetta fragment assembly method was selected for performing the modeling study. The homology model of NSP6 of SARS-CoV-2 obtained and is depicted in Figure S3a. The Ramachandran plot which was used for the validation of the homology model retrieved using PROCHECK server shows that the homology model of NSP6 of SARS-CoV-2 shows that nearly $95.1 \%$ of the residues are in the favored region (Figure S3b). Moreover, ERRAT score for the NSP4 homolog model was 97.1326 which indicates the model is good for further studies.

\section{Molecular docking studies of NSP6 of SARS-CoV-2 against the FDA-approved compounds}

1615 FDA-approved compounds were made to dock with NSP6 homology model of SARS-CoV-2 using the GLIDE module of Schrodinger. Sucrose (ZINC000004217475) was found to have high affinity against NSP6 and it had a docking score of $-7.908 \mathrm{kcal} / \mathrm{mol}$. Interactions of Sucrose with the modeled NSP6 are depicted in Fig. 12, respectively.

\section{Molecular dynamics simulation analysis of NSP6-Sucrose docked complex}

Molecular dynamics simulations of NSP6 was performed and the RMSD obtained reveal that NSP6 was almost stable around $0.4 \mathrm{~nm}$ and remained the same till the end of simulation (Fig. 3). Rg graph of NSP6 was stable throughout the dynamics (Fig. 4). RMSF graph of NSP6 reveals residues 77-101 consist of a helix-loop-helix; residues 233-258 also consist of a loop and a helix having fluctuations above $0.2 \mathrm{~nm}$ (Fig. 13). These regions having higher fluctuations and are near the ligand-binding site and help in ligand interaction. The hydrogen bonds obtained through dynamics between NSP6 and Sucrose are depicted in Fig. 14.

\section{Protein-protein docking studies of NSP6 with sigma-1 receptor}

Protein-protein docking studies of NSP6 with Sigma-1 receptor were carried out using Cluspro server. Interactions of NSP6 with Sigma-1 receptor are depicted in Fig. 15. From the interaction figures of Sigma-1 receptor and Sucrose with NSP6, we found that both of them bound to the same binding site of NSP6.
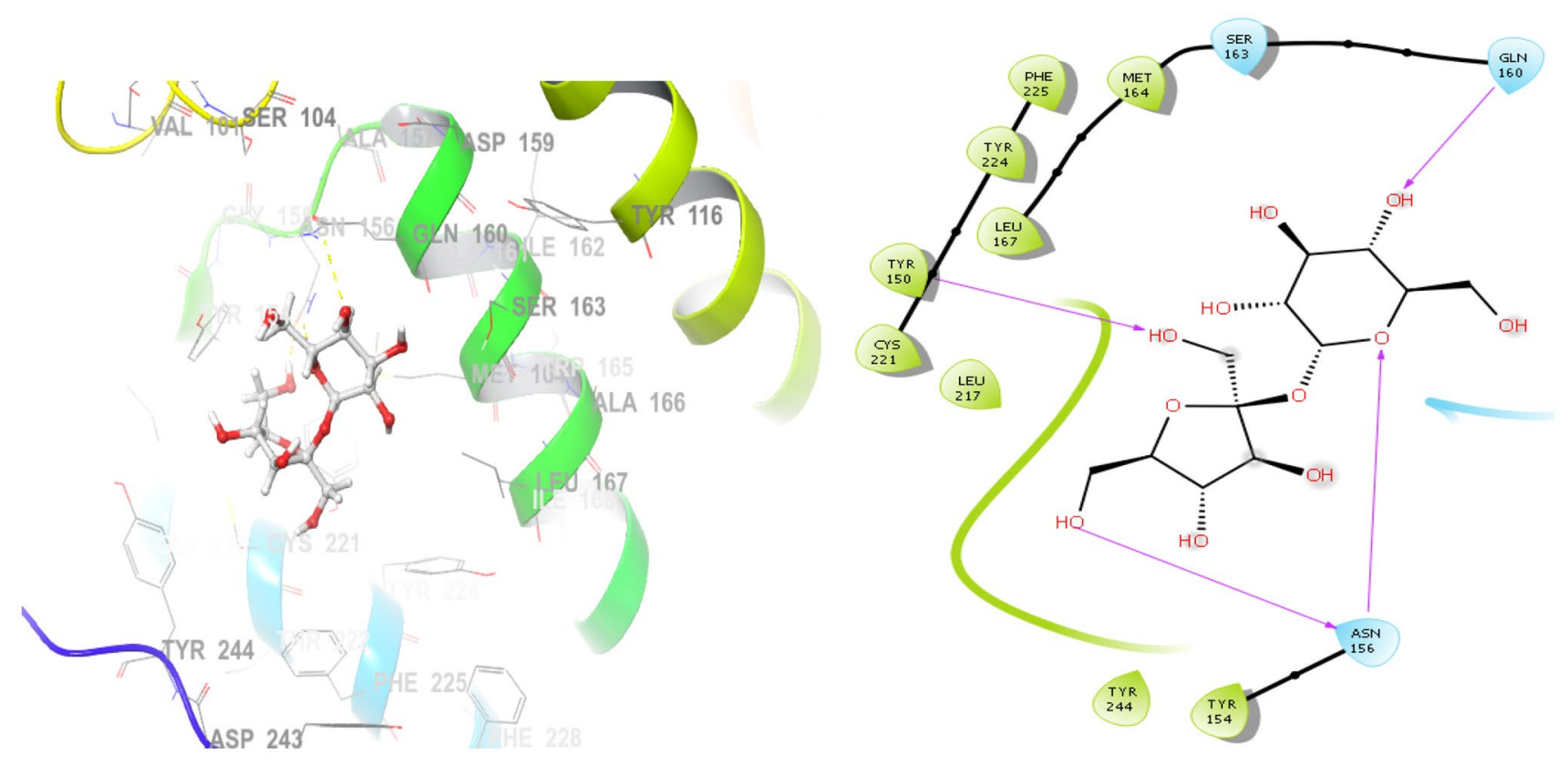

Fig. 12 Sucrose interactions with modeled NSP6. Hydrogen bonds between NSP6 and Sucrose are depicted in the figures 


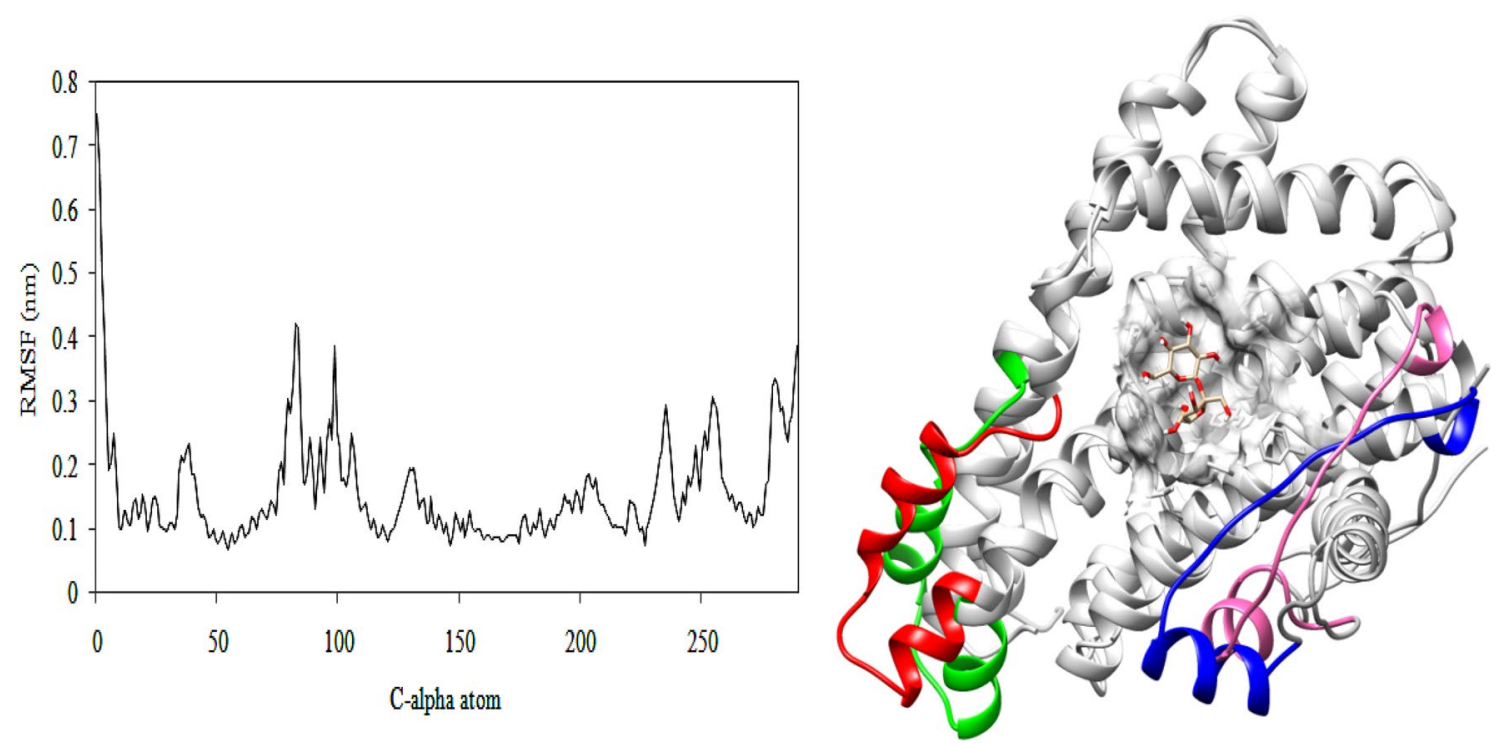

Fig. 13 Fluctuations of NSP6 residues during dynamics. Superimposed figure of NSP6 at 0 ns and at 25 ns, dynamics of the residues 77-101 (0th ns in red; 25th ns in green) is shown, residues 233-258 (0th ns in blue; 25th ns in pink). Sucrose is represented as atoms

\section{Non-structural protein 13 (NSP13) of SARS-CoV-2}

NSP13 acts as a helicase and plays a vital role in catalyzing the unwinding of duplex oligonucleotides into single strands. Yuen et al. have established that NSP13, NSP14, NSP15 and orf6 function as potent interferon antagonists (Yuen et al. 2020). Importantly, NSP13 has been recognized as an ideal drug target for the development of anti-viral drugs due to its high sequence similarity across all $\mathrm{CoV}$ species (Jia et al. 2019). A recent study through integrative virtual screening and molecular dynamics simulations approach has identified

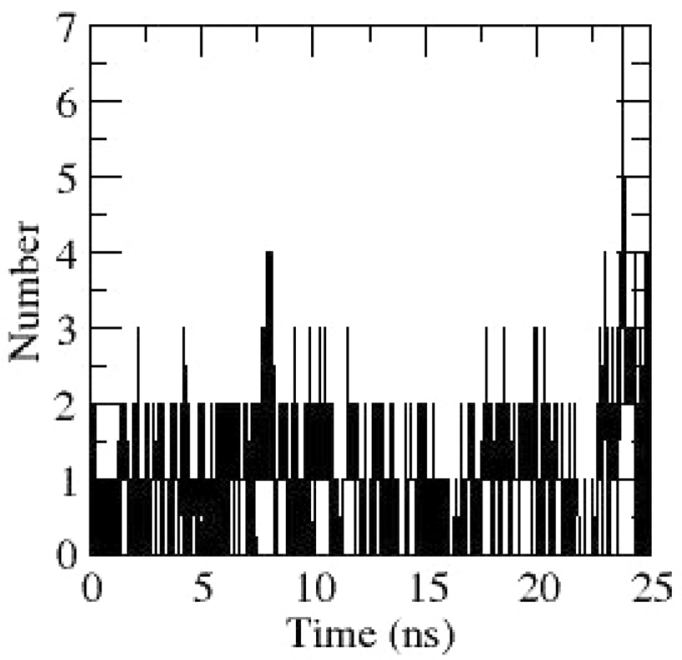

Fig. 14 Hydrogen bonds between NSP6 and Sucrose obtained during dynamics potential binding modes and favorable molecular interaction profile of certain compounds against NSP13 (Mirza and Froeyen 2020). Another study established that NTPase and helicase activities can be inhibited by bismuth salts (Shu et al. 2020). Furthermore, studies reveal that NSP12 enhances the helicase activity of NSP13 through a direct protein-protein interaction $^{25}$.

\section{Homology modeling of NSP13 of SARS-CoV-2}

FASTA sequence of NSP13 of SARS-CoV-2 was retrieved from Uniprot (ID: P0DTD1, residues 5325-5921(1-596)) and was modeled using SWISS-MODEL server. The 3D structure of SARS-CoV NSP13 (PDB ID: 6JYT) which bears $99.83 \%$ sequence identity to the NSP13 of SARS$\mathrm{CoV}-2$ was used as the template for homology modeling. The homology model of NSP13 of SARS-CoV-2 obtained and is depicted in Figure S4a. The Ramachandran plot which was used for the validation of the homology model retrieved using PROCHECK server shows that the homology model of NSP13 of SARS-CoV-2 shows that nearly $80.3 \%$ of the residues are in the most favored region (Fig. S4b). While preparing this manuscript, Newman et al. have published the 3D protein structure of NSP13 (PDB ID: 6ZSL). The RMSD was computed between the homology model and the 3D structure of NSP13 was around $0.9 \AA$. RMSD values less than $3 \AA$ can be considered for closely homologous proteins (Reva et al. 1998). Moreover, ERRAT score for the NSP4 homolog model was 75.6757 which indicates the model is good for further studies. 


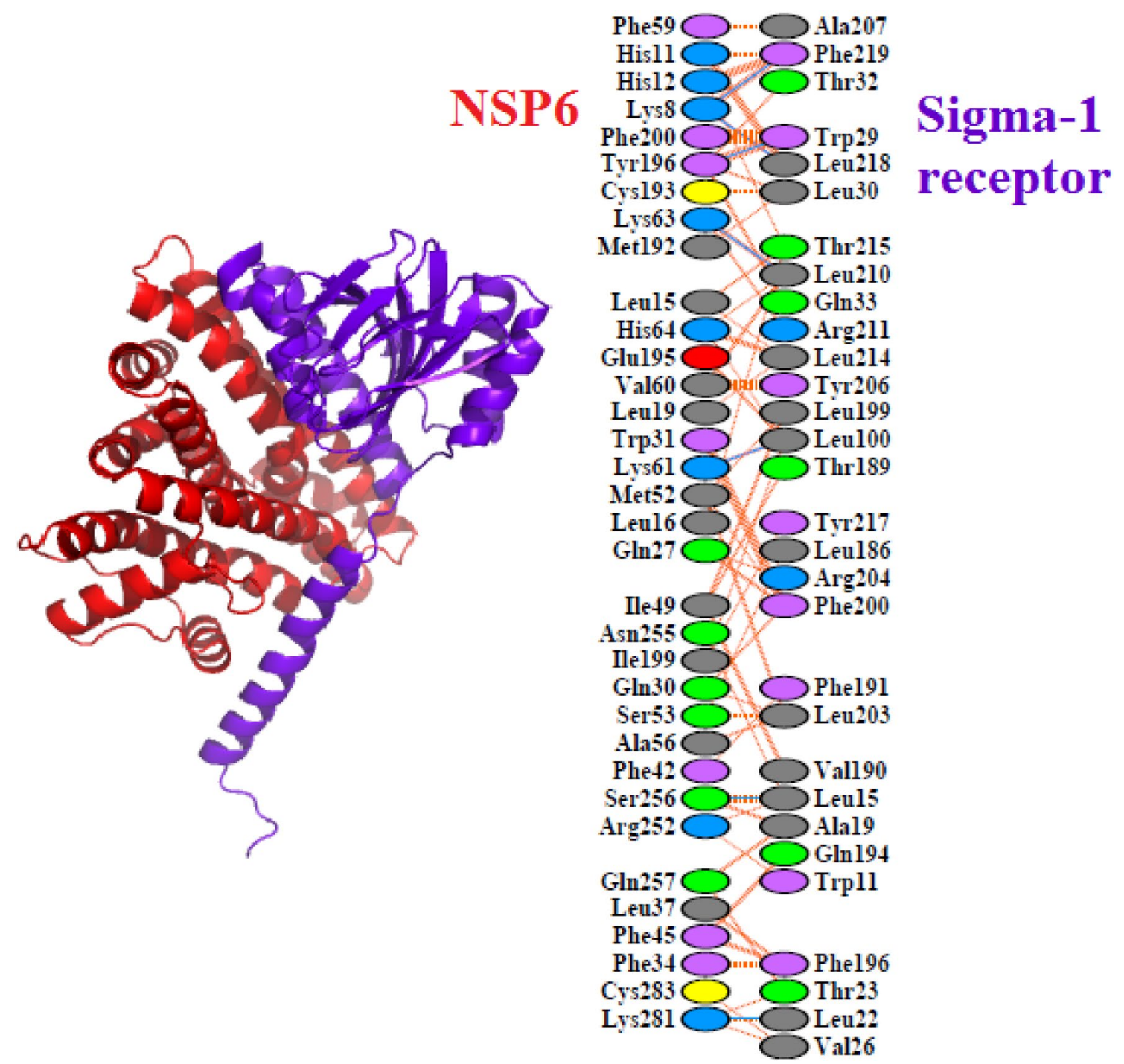

Fig. 15 Protein-protein molecular docking studies of NSP6 with Sucrose. Interactions between NSP6 and Sucrose are also depicted

\section{Molecular docking studies of NSP13 of SARS-CoV-2 against the FDA-approved compounds}

1615 FDA-approved compounds were made to dock with NSP13 of SARS-CoV-2 using the GLIDE module of Schrodinger. Acarbose (ZINC000087496092) was found to have high affinity against NSP13 and it had a docking score of $-10.73 \mathrm{kcal} / \mathrm{mol}$. Acarbose is an inhibitor of alpha glucosidase and it acts by retarding the digestion and absorption of carbohydrates in the small intestine. It is given orally to non-insulin-dependent diabetes mellitus patients for reducing the increase in blood-glucose concentrations (Chiasson et al. 2002). Acarbose can be preferred for treating COVID19 patients with uncontrollable diabetes. Interactions of Acarbose with the modeled NSP13 are depicted in Fig. 16, respectively.

\section{Molecular dynamics simulation analysis of NSP13- Acarbose docked complex}

MD simulation of NSP13-Acarbose docked complex was performed for $25 \mathrm{~ns}$. RMSD of NSP13 obtained during dynamics is stable around $0.5 \mathrm{~nm}$ from $15 \mathrm{~ns}$ till the end of simulation and is depicted in Fig. 3. Rg values of NSP13 decrease from $2.8 \mathrm{~nm}$ to $2.7 \mathrm{~nm}$, which indicates that NSP13 is trying to attain a compact structure during dynamics (Fig. 4). From the RMSF plot of NSP13 (Fig. 17), regions having fluctuations greater than $0.4 \mathrm{~nm}$ are considered significant. Residues 50-79, 497-504 of NSP13 have significantly higher fluctuations during dynamics. However, the importance of these residues is unresolved. The hydrogen bonding between NSP13 and Acarbose obtained through dynamics is given in Fig. 18. 


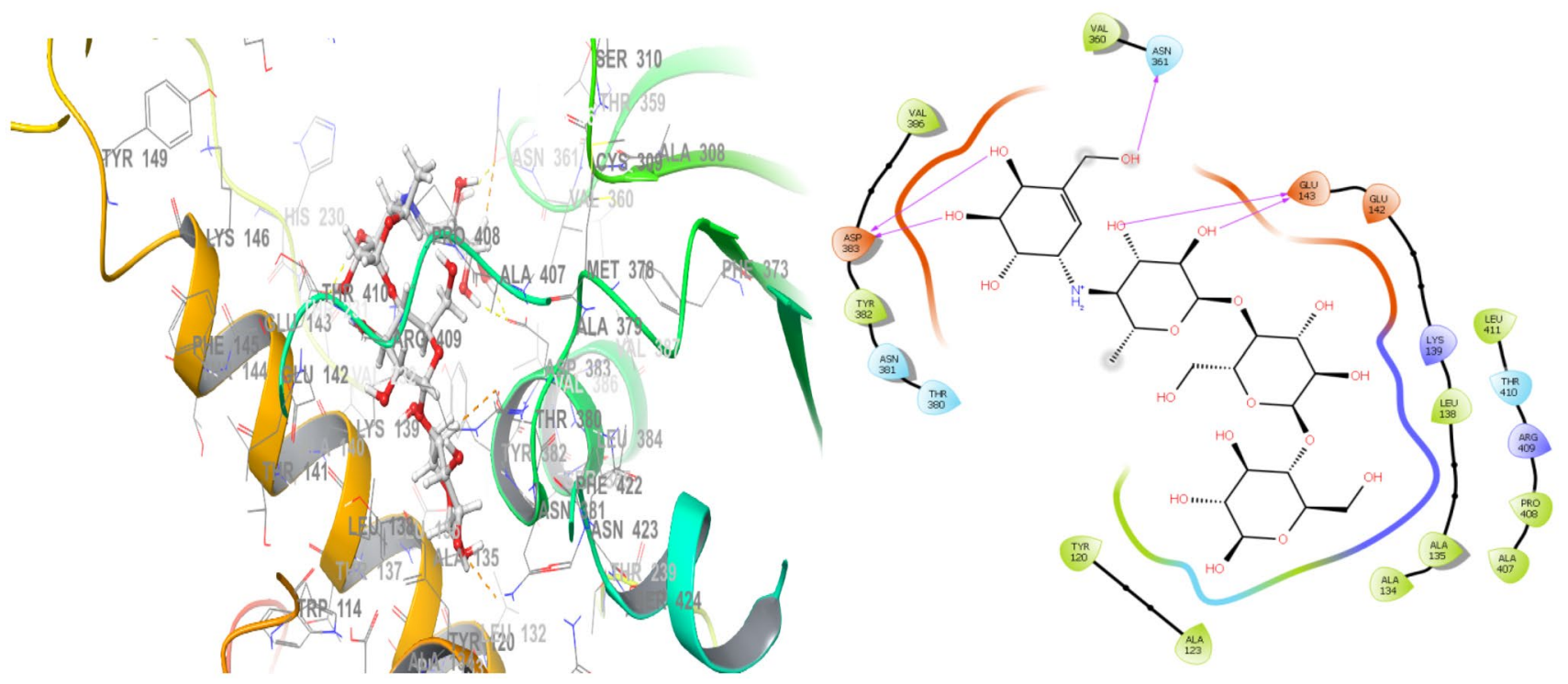

Fig. 16 Acarbose interactions with modeled NSP13. Hydrogen bonds between NSP13 and Acarbose are depicted in the figures
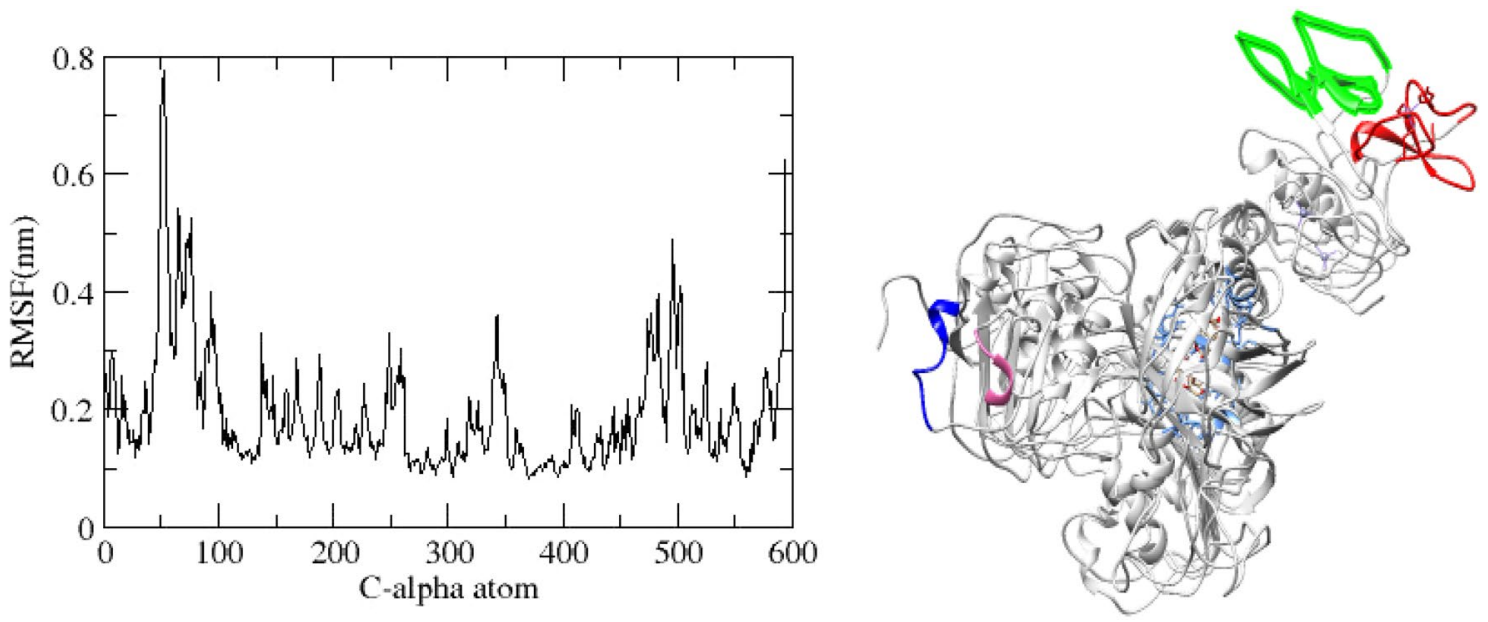

Fig. 17 Fluctuations of NSP13 residues during dynamics. Superimposed figure of NSP13 at $0 \mathrm{~ns}$ and at $25 \mathrm{~ns}$, dynamics of the residues 50-79 (0th ns in red; 25th ns in green) is shown, residues 497-504

\section{Protein-protein molecular docking studies of NSP13 with TBK1}

Protein-protein docking studies of NSP13 with Insulindegrading enzyme TANK-binding kinase 1 (TBK1) were carried out using Cluspro server. Interactions of NSP13 with TBK1 are depicted in Fig. 19. From the interaction figures of TBK1 and Acarbose, we found that both of them bound to the same binding site of NSP13. (0th ns in blue; 25th ns in pink). Acarbose is represented as atoms. Acarbose-binding residues are highlighted in light blue

\section{Retrieval of physiochemical properties of the top-hit ligands against the NSPs}

Further, physiochemical properties of the top-scored compounds inhibiting the NSPs are retrieved using the SwissADME webserver (Daina et al. 2017) and is listed in Table 1.

From in silico studies, Lima and Silva (2020) have identified three potential compounds, namely Tirilazad, 


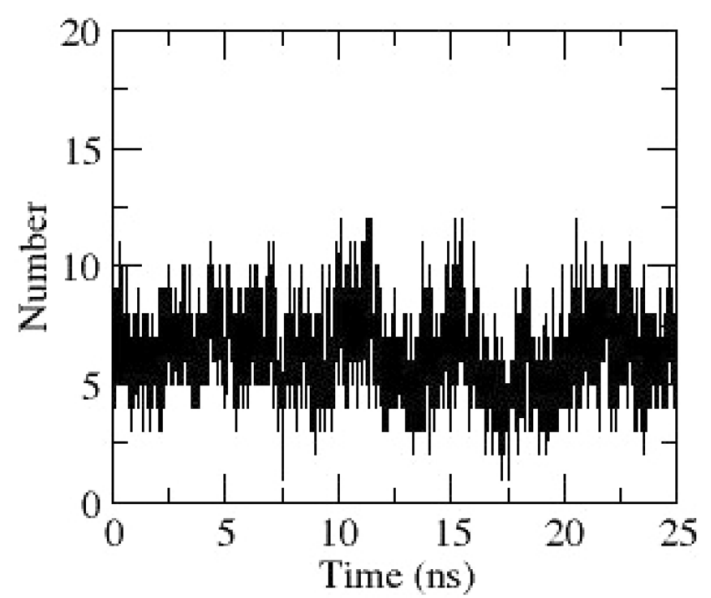

Fig. 18 Hydrogen bonds between NSP13 and Acarbose obtained through dynamics
Phthalocyanine and Zk-806450 with better affinity scores (in the range of -8 to $-9 \mathrm{kcal} / \mathrm{mol}$ ) against NSP1 of SARSCoV-2. From our study, we have identified a potential antibiotic and a FDA-approved drug Ceftolozane with similar binding energy of $-8 \mathrm{kcal} / \mathrm{mol}$ for NSP1, since Ceftolozane is readily available and it can be used for inhibition of NSP1 and thereby enhances the treatment of COVID-19 patients. Meshari and Motwalli (2020) through molecular docking studies have reported Baicalin, a natural compound to be moderately effective (binding energy in the range of $-4 \mathrm{kcal} / \mathrm{mol}$ ) against NSP4 of SARS-CoV-2. However, we report a FDA-approved drug Amphotericin B which has better binding energy $(-9 \mathrm{kcal} / \mathrm{mol})$ than Baicalin, consequently can be very effective in inhibiting NSP4. Moreover for NSP13, Mirza and Froeyen $2020^{25}$ have identified potential compounds inhibiting the helicase. Although, we

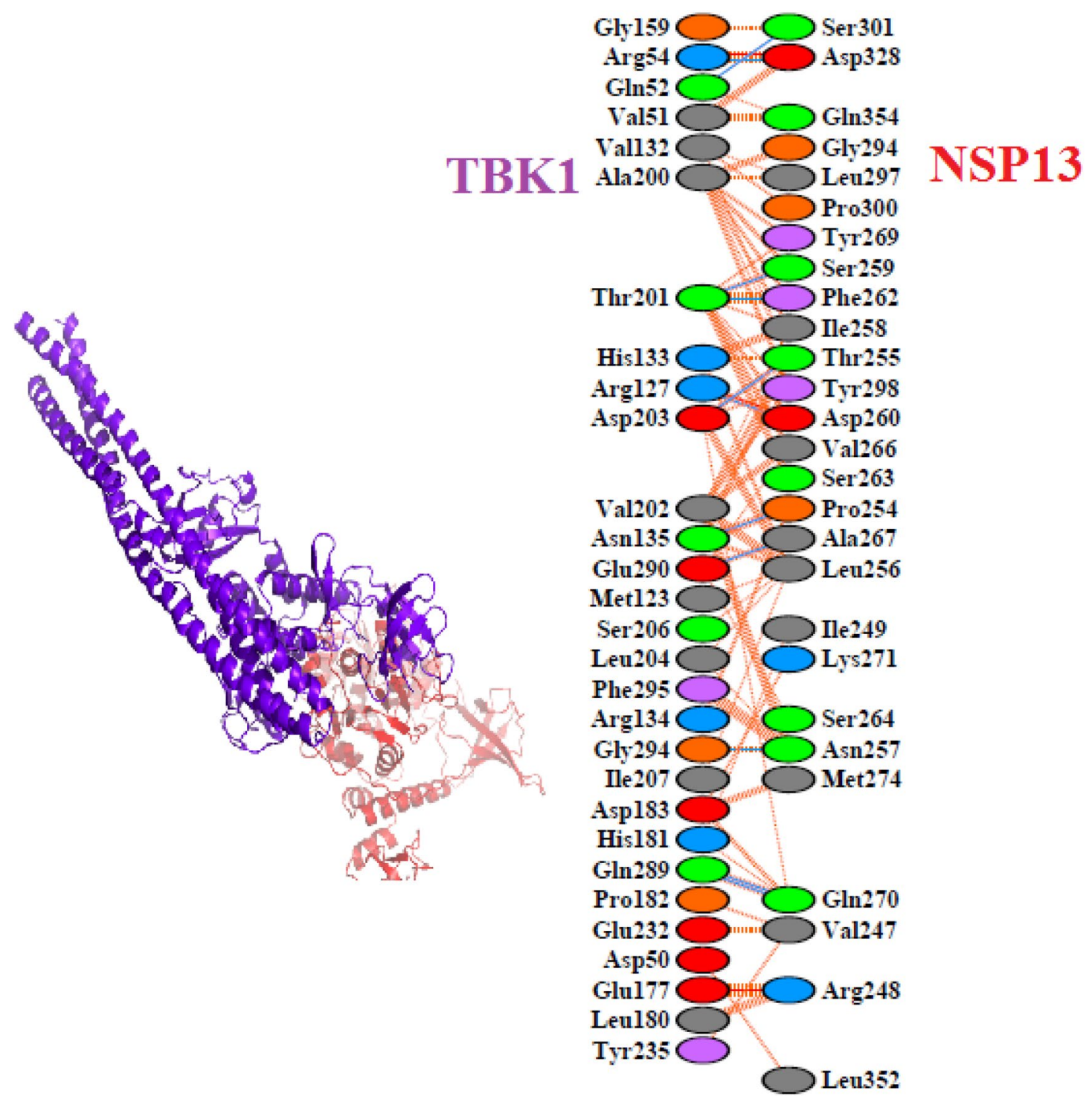

Fig. 19 Protein-protein molecular docking studies of NSP13 with TBK1. Interactions between NSP13 and TBK1 are also depicted 
Table 1 Physiochemical properties of the top-hit ligands retrieved during the study

\begin{tabular}{|c|c|c|c|}
\hline \multirow{2}{*}{$\frac{\text { NSPs }}{\text { NSP1 }}$} & \multirow{2}{*}{$\frac{\text { Top-hit ligands }}{\text { Ceftolozane }}$} & \multicolumn{2}{|c|}{ Physiochemical properties } \\
\hline & & Formula & $\mathrm{C} 23 \mathrm{H} 30 \mathrm{~N} 12 \mathrm{O} 8 \mathrm{~S} 2$ \\
\hline & & Molecular weight & $666.69 \mathrm{~g} / \mathrm{mol}$ \\
\hline & & Num. heavy atoms & 45 \\
\hline & & $\begin{array}{l}\text { Num. arom. heavy } \\
\text { atoms }\end{array}$ & 10 \\
\hline & & Fraction Csp3 & 0.43 \\
\hline & & Num. rotatable bonds & 15 \\
\hline & & $\begin{array}{l}\text { Num. H-bond accep- } \\
\text { tors }\end{array}$ & 12 \\
\hline & & Num. H-bond donors & 7 \\
\hline & & Molar Refractivity & 162.87 \\
\hline & & Log Po/w (XLOGP3) & -3.84 \\
\hline & & $\log \mathrm{S}(\mathrm{ESOL})$ & -0.73 \\
\hline \multirow[t]{11}{*}{ NSP4 } & Amphotericin B & Formula & C47H73NO17 \\
\hline & & Molecular weight & $924.08 \mathrm{~g} / \mathrm{mol}$ \\
\hline & & Num. heavy atoms & 65 \\
\hline & & $\begin{array}{l}\text { Num. arom. heavy } \\
\text { atoms }\end{array}$ & 0 \\
\hline & & Fraction Csp3 & 0.66 \\
\hline & & Num. rotatable bonds & 3 \\
\hline & & $\begin{array}{l}\text { Num. H-bond accep- } \\
\text { tors }\end{array}$ & 18 \\
\hline & & Num. H-bond donors & 12 \\
\hline & & Molar Refractivity & 239.06 \\
\hline & & Log Po/w (XLOGP3) & 0.00 \\
\hline & & $\log \mathrm{S}(\mathrm{ESOL})$ & -5.37 \\
\hline \multirow[t]{11}{*}{ NSP6 } & Sucrose & Formula & $\mathrm{C} 12 \mathrm{H} 22 \mathrm{O} 11$ \\
\hline & & Molecular weight & $342.30 \mathrm{~g} / \mathrm{mol}$ \\
\hline & & Num. heavy atoms & 23 \\
\hline & & $\begin{array}{l}\text { Num. arom. heavy } \\
\text { atoms }\end{array}$ & 0 \\
\hline & & Fraction Csp3 & 1.00 \\
\hline & & Num. rotatable bonds & 5 \\
\hline & & $\begin{array}{l}\text { Num. H-bond accep- } \\
\text { tors }\end{array}$ & 11 \\
\hline & & Num. H-bond donors & 8 \\
\hline & & Molar Refractivity & 68.16 \\
\hline & & $\log P_{\mathrm{o} / \mathrm{w}}(\mathrm{XLOGP} 3)$ & -3.70 \\
\hline & & $\log S(\mathrm{ESOL})$ & 0.70 \\
\hline
\end{tabular}

Table 1 (continued)

\begin{tabular}{llll}
\hline NSPs & Top-hit ligands & \multicolumn{2}{l}{ Physiochemical properties } \\
\hline NSP13 & Acarbose & Formula & C25H43NO18 \\
& Molecular weight & $645.60 \mathrm{~g} / \mathrm{mol}$ \\
& Num. heavy atoms & 44 \\
& Num. arom. heavy & 0 \\
& atoms & \\
& Fraction Csp3 & 0.92 \\
& Num. rotatable bonds & 9 \\
& Num. H-bond accep- & 19 \\
& tors & \\
& Num. H-bond donors & 14 \\
& Molar Refractivity & 136.69 \\
& Log $P_{\text {o/w }}$ (XLOGP3) & -8.53 \\
& Log $S$ (ESOL) & 2.13 \\
&
\end{tabular}

have identified a better compound Acarbose binging with higher affinity (in the range of $-11 \mathrm{kcal} / \mathrm{mol}$ ) compared to those compounds identified by Mirza and Froeyen (2020). Moreover, Acarbose is a FDA-approved drug and has better physiochemical properties. Hence, this study results new and better compounds against these four less explored NSP proteins and, thus, further enhance the COVID-19 treatment.

\section{Conclusion}

COVID-19 pandemic has become a global emergency and there is an urgent need to identify effective drugs, with lower side effects to fight against SARS-CoV-2. Therefore, in this study, we conducted a virtual screening of 1615 FDA-approved drugs targeting four non-structural proteins (NSP1, NSP4, NSP6 and NSP13) of SARS-CoV-2. Studies on these four NSPs are relatively obscure. We have modeled the three-dimensional structures for these four NSPs using online servers. From our molecular docking studies, we have identified potential compounds which could be used to inhibit these proteins and be used for treating COVID-19 patients. Molecular dynamics studies were performed for the protein-ligand complexes to study the interactions in detail. Thus, the insights gained from this study will be supportive in treatment of COVID-19 patients.

Supplementary Information The online version contains supplementary material available at https://doi.org/10.1007/s42485-021-00067-w.

Acknowledgements The authors thank the DBT-Bioinformatics center at the Department of Bioinformatics, Bharathiar University for the computational facilities. Shobana Sundar acknowledges the financial 
support received through the award of ICMR-Research Associate (Award Letter No: ISRM/11(13)/2019). Lokesh Thangamani acknowledges the financial support received through the award of ICMR-Senior Research Fellowship (Award Letter No: BMI/11(01)/2020)

\section{Declarations}

Conflict of interest All the authors declare that they have no conflict of interest.

\section{References}

Abraham MJ, Murtola T, Schulz R, Páll S, Smith JC, Hess B, Lindahl E (2015) GROMACS: High performance molecular simulations through multi-level parallelism from laptops to supercomputers. SoftwareX. 1:19-25

Benvenuto D, Angeletti S, Giovanetti M, Bianchi M, Pascarella S, Cauda R, Ciccozzi M, Cassone A (2020) Evolutionary analysis of SARS-CoV-2: how mutation of non-structural protein 6 (NSP6) could affect viral autophagy. J Infect 81(1):e24-e27

Cao B, Wang Y, Wen D, Liu W, Wang J, Fan G, Ruan L, Song B, Cai Y, Wei M, Li X (2020) A trial of lopinavir-ritonavir in adults hospitalized with severe Covid-19. N Engl J Med

Chen Y, Liu Q, Guo D (2020) Emerging coronaviruses: genome structure, replication, and pathogenesis. J Med Virol 92(4):418-423

Chiasson JL, Josse RG, Gomis R, Hanefeld M, Karasik A, Laakso M, STOP-NIDDM Trial Research Group (2002) Acarbose for prevention of type 2 diabetes mellitus: the STOP-NIDDM randomised trial. The Lancet. 359(9323):2072-7

Clark LK, Green TJ, Petit CM (2021) Structure of nonstructural protein 1 from SARS-CoV-2. J Virol 95(4)

Daina A, Michielin O, Zoete V (2017) SwissADME: a free web tool to evaluate pharmacokinetics, drug-likeness and medicinal chemistry friendliness of small molecules. Sci Rep 7(1):1-3

de Lima Menezes G, da Silva RA (2020) Identification of potential drugs against SARS-CoV-2 non-structural protein 1 (nsp1). J Biomol Struct Dyn:1-1.

de Lima Menezes G, da Silva RA (2020) Identification of potential drugs against SARS-CoV-2 non-structural protein 1 (nsp1). J Biomol Struct Dyn. 1-1.

Gil C, Ginex T, Maestro I, Nozal V, Barrado-Gil L, Cuesta-Geijo MÁ, Urquiza J, Ramírez D, Alonso C, Campillo NE, Martinez A (2020) COVID-19: drug targets and potential treatments. J Med Chem 63(21):12359-12386

Gordon DE, Jang GM, Bouhaddou M, Xu J, Obernier K, White KM, O'Meara MJ, Rezelj VV, Guo JZ, Swaney DL, Tummino TA (2020) A SARS-CoV-2 protein interaction map reveals targets for drug repurposing. Nature. 583(7816):459-68.

Hosseini M, Chen W, Xiao D, Wang C (2020) Computational molecular docking and virtual screening revealed promising SARSCoV-2 drugs. Precision Clin Med

Huang C, Lokugamage KG, Rozovics JM, Narayanan K, Semler BL, Makino S (2011) SARS coronavirus NSP1 protein induces template-dependent endonucleolytic cleavage of mRNAs: viral mRNAs are resistant to NSP1-induced RNA cleavage. PLoS Pathog 7(12):e1002433

Irwin JJ, Shoichet BK (2005) ZINC-a free database of commercially available compounds for virtual screening. J Chem Information Modeling. 45(1):177-182
Jia Z, Yan L, Ren Z, Wu L, Wang J, Guo J, Zheng L, Ming Z, Zhang L, Lou Z, Rao Z (2019) Delicate structural coordination of the severe acute respiratory syndrome coronavirus Nsp13 upon ATP hydrolysis. Nucleic Acids Res 47(12):6538-6550

Laskowski RA, MacArthur MW, Thornton JM (2006) PROCHECK: validation of protein-structure coordinates.

Lemke A, Kiderlen AF, Kayser O (2005) Amphotericin b. Appl Microbiol Biotechnol 68(2):151-162

Meshari A, Motwalli O (2020) In silico virtual screening, characterization, docking and molecular dynamics studies of crucial SARS-CoV-2 proteins. J Biomol Struct Dyn 1-11

Mirza MU, Froeyen M (2020) Structural elucidation of SARS-CoV-2 vital proteins: Computational methods reveal potential drug candidates against main protease, Nsp12 polymerase and Nsp13 helicase. J Pharmaceutical Anal 10(4):320-328

Narayanan K, Huang C, Lokugamage K, Kamitani W, Ikegami T, Tseng CTK, Makino S, Narayanan K, Huang C, Lokugamage K, Kamitani W, Ikegami T, Tseng CT, Makino S (2008) Severe acute respiratory syndrome coronavirus nsp1 suppresses host gene expression, including that of type I interferon, in infected cells. J Virol 82(9):4471-4479

Reva BA, Finkelstein AV, Skolnick J (1998) What is the probability of a chance prediction of a protein structure with an rmsd of 6 å? Folding Design 3(2):141-147

Rohl CA, Strauss CE, Misura KM, Baker D (2004) Protein structure prediction using Rosetta. Methods Enzymol 383:66-93

Sakai Y, Kawachi K, Terada Y, Omori H, Matsuura Y, Kamitani W (2017) Two-amino acids change in the nsp4 of SARS coronavirus abolishes viral replication. Virology 510:165-174

Schwede T, Kopp J, Guex N (2003) Peitsch MC (2003) SWISSMODEL: an automated protein homology-modeling server. Nucleic Acids Res 31(13):3381-3385

Sharma A, Tiwari V, Sowdhamini R (2020) Computational search for potential COVID-19 drugs from FDA-approved drugs and small molecules of natural origin identifies several anti-virals and plant products. J Biosci 45(1):1-8

Shu T, Huang M, Wu D, Ren Y, Zhang X, Han Y, Mu J, Wang R, Qiu Y, Zhang DY, Zhou X (2020) SARS-Coronavirus-2 Nsp13 possesses NTPase and RNA helicase activities that can be inhibited by bismuth salts. Virologica Sinica. 35:321-329

van Duin D, Bonomo RA (2016) Ceftazidime/avibactam and ceftolozane/tazobactam: second-generation $\beta$-lactam/ $\beta$-lactamase inhibitor combinations. Clin Infect Dis 63(2):234-241

Wang M, Cao R, Zhang L, Yang X, Liu J, Xu M, Shi Z, Hu Z, Zhong W, Xiao G (2020) Remdesivir and chloroquine effectively inhibit the recently emerged novel coronavirus (2019-nCoV) in vitro. Cell Res 30(3):269-271

World Health Organization, 2020

Xu X, Lou Z, Ma Y, Chen X, Yang Z, Tong X, Zhao Q, Xu Y, Deng H, Bartlam M, Rao Z (2009) Crystal structure of the C-terminal cytoplasmic domain of non-structural protein 4 from mouse hepatitis virus A59. PLoS ONE 4(7):e6217

Yuen CK, Lam JY, Wong WM, Mak LF, Wang X, Chu H, Cai JP, Jin DY, To KK, Chan JF, Yuen KY (2020) SARS-CoV-2 nsp13, nsp14, nsp15 and orf6 function as potent interferon antagonists. Emerging Microbes Infect 9(1):1418-1428

Publisher's Note Springer Nature remains neutral with regard to jurisdictional claims in published maps and institutional affiliations. 